\title{
Model-free model-fitting and predictive distributions REJOINDER
}

\author{
Dimitris N. Politis
}

I am sincerely grateful to all discussants for their an in-depth reading of the paper, and for sharing their insights on extensions and applications to quite diverse areas.

The discussants raise a number of interesting points. I am particularly thankful to Stefan Sperlich for such a tour-de-force of potential Model-free Prediction applications to challenging problems in Biostatistics, Econometrics, and even Environmetrics. His MF/MB treatment of the important class of Mixed Effect Models - and the related small area estimatorsis crystal-clear. In addition, there are many open problems mentioned in his report; future work may hopefully shed light on some of them. To focus on the simple random intercept model (1), it may also be possible to construct an extension of the $\mathrm{MF}^{2}$ (or even $\mathrm{MF} / \mathrm{MF}^{2}$ ) procedure by (a) working out the MF procedure and producing $Y^{*}$ pseudo-values for each value of $d$ separately; and (b) use the technique of Lombardia and Sperlich (2008) to employ an estimated conditional (on $x$ ) distribution in order to to generate a pseudo-intercept $u_{d}^{*}$ that is to be added to $Y^{*}$.

I would like to especially thank Juan Cuesta-Albertos for his many constructive suggestions over the last couple of years that helped clear some difficult issues both conceptual as well as practical. He indicates a preference for the $\mathrm{MF}^{2}$ procedure of Remark 4.5, i.e., generating $u_{1}^{*}, \ldots, u_{n}^{*} \sim$ i.i.d. from an exact Uniform $(0,1)$ as opposed to resampling the quasi-residuals $u_{1}, \ldots, u_{n}$; indeed, this may have some advantages. The main advantage, in my opinion, is that the quantile inverse $\hat{D}_{x_{\mathrm{f}}}^{-1}$ can then directly be used to obtain the bootstrap data although $\hat{D}_{x_{\mathrm{f}}}$ is a step function, and the step of producing a smooth estimator of $D_{x_{\mathrm{f}}}(y)$ as a function of $y$ is not needed. In this way, the $\mathrm{MF}^{2}$ procedure of Remark 4.5 is seen to be applicable even in case the true $D_{x}(y)$ is not continuous in $y$ resulting in something like a "Model-free" GLM (Generalized Linear Model).

Nevertheless, there are also two potential disadvantages:

(a) It is in the spirit of bootstrap methods to try to use the original data as much as possible in the hope that the bootstrap world would mimic the real world closely. There are examples, such as the wild bootstrap, where the practitioner may benefit from 
sampling using artificial distributions; but even in the wild bootstrap, the magnitude of the original residuals is retained, and plays a crucial role in the procedure.

(b) Using $u_{i}^{*} \sim$ i.i.d. Uniform $(0,1)$ does not allow us to construct the extension of the $\mathrm{MF}^{2}$ procedure that is called $\mathrm{MF} / \mathrm{MF}^{2}$ in Section 4.5. The delete-one quasi-residuals $u_{t}^{(t)}$ are expected to be a bit further away from their mean than the exact uniform distribution would dictate; this slight non-uniformity of the $u_{t}^{(t)}$ is a finite-sample effect that leads to wider predictive intervals as in the MF/MB case.

Because of the above, my personal preference is indeed to resample the original $u_{t}$ (or $u_{t}^{(t)}$ ) quasi-residuals - as long as $D_{x_{\mathrm{f}}}(y)$ is smooth as a function of $y$. However, I promise to look into the matter again in future work in order to further compare the two options.

Ingrid Van Keilegom brings up the issue of possible endogeneity; this is a challenging and 'hot' topic in current econometric research - see e.g. all the recent literature on instrumental variables. In fact, endogeneity appears to be an underlying issue in some of the problems discussed by Sperlich. My paper focuses on the regressors being deterministic both for simplicity of exposition but also in order to better grasp the problem at hand, i.e., prediction of $g\left(Y_{\mathrm{f}}\right)$ that corresponds to the regressor taking value equal to some real number $x_{\mathrm{f}}$. If the regressors are random, does this question make sense as stated? The answer is yes, and there is often an underlying time element. For example, let $X$ denote the number of available houses for sale in a given zip code, and $Y$ be the median house price three months later; the law of supply and demand would suggest that $Y$ is inversely related to $X$. If one observes $X=x_{\mathrm{f}}$, it is then natural to want to predict $Y_{\mathrm{f}}$ since its value would not be known for another three months!

With regressors that are random but independent of the error term, the model-based and $\mathrm{MF} / \mathrm{MB}$ methods in the paper seem to apply verbatim with the usual understanding of inference carried out conditionally on the observed values for the regressors (including $X=x_{\mathrm{f}}$ ). In the presence of endogeneity, however, things are a bit different. Since the regressor is correlated with the error term, the usual model-based (and MF/MB) reasoning breaks down. Nevertheless, the MF viewpoint of Section 4 does not require an additive model. So the only issue here is to be able to identify the form of the optimal predictor since the conditional expectation $E\left(g\left(Y_{\mathrm{f}}\right) \mid X=x_{\mathrm{f}}\right)$ is no longer appropriate. If the form of the optimal predictor is known (or can be approximated), then it should be used as the value of $\Pi$ and $\Pi^{*}$ in the construction of the prediction roots in the real and in the bootstrap world respectively; with this single modification, it appears that the $\mathrm{MF}^{2}$ and $\mathrm{MF} / \mathrm{MF}^{2}$ prediction intervals would remain valid as stated.

Still in the MF setup of Section 4, Carlos Velasco suggests that instead of focusing on the conditional distribution of $Y$ given $X$ in order to predict $g\left(Y_{\mathrm{f}}\right)$ given $X=x_{\mathrm{f}}$, we could 
just as well define $W=g(Y)$, and use the conditional distribution of $W$ given $X$ to predict $W_{\mathrm{f}}$ given $X=x_{\mathrm{f}}$ where $W_{\mathrm{f}}=g\left(Y_{\mathrm{f}}\right)$. This is, of course, fine as long as regularity conditions are maintained. To give an example where they are not, consider the cps71 wage vs. age dataset shown in Figure 3, and suppose the question is to predict whether a particular 50-year old man will earn a salary over a given threshold, say $A$. Then, $g(y)=\mathbf{1}\{y>A\}$, and because of its $0-1$ nature, the conditional distribution of $W=g(Y)$ is not continuous. Hence, the MF approach focusing on the conditional distribution of $W$ given $X$ would not work - unless the aforementioned trick is used, i.e., using the $\mathrm{MF}^{2}$ procedure of Remark 4.5 to allow for the discreteness. By contrast, the MF approach focusing on the conditional distribution of $Y$ given $X$ works regardless of the nature of function $g$, and that includes the $\mathrm{MF} / \mathrm{MF}^{2}$ variant.

As Velasco further notes, the MF ideas may find application in time series prediction where the issue of conditioning on the last $p$ (say) observed values enters. I can not do justice to this difficult problem in such a short write-up but this gives me the opportunity to refer to a forthcoming manuscript on prediction intervals for autoregressions and Markov processes that is joint work with my Ph.D. student Li Pan.

Manuel Febrero-Bande indicates that he obtained good results with the local bootstrap of Shi (1991); this is a powerful resampling method for regression with extensions to other fields - see e.g. Paparoditis and Politis (1999). As he correctly notes, the local bootstrap is closer to the $\mathrm{MF}^{2}$ ideas of Section 4 since, in a model-based setting, the residuals should be close to i.i.d. and not require 'windowing' - to borrow a phrase from Härdle and Marron (1991). I hope to be able to compare the local bootstrap with the $\mathrm{MF}^{2}$ procedures of Section 4 in future work.

Febrero-Bande further suggests potential applications of Model-free Prediction to Functional Data Analysis (FDA). Indeed, this is a very exciting prospect. The case where the response $Y$ is real-valued and the regressor takes values in a function space seems quite straightforward due to work such as Cai and Hall (2006) for linear regression, and the Toulouse school regarding FDA kernel smoothing - see Ferraty and Vieu (2006) and the references therein.

Finally, several discussants are interested in asymptotic results; it is true that there is little by way of asymptotic theory in the paper both because of lack of space but mainly because the issue of asymptotic validity and accuracy of prediction intervals does not seem to be generally well understood. To elaborate, the usual definition for a prediction interval to be called asymptotically valid is that its coverage probability tends to the nominal as $n \rightarrow \infty$; see e.g. Schmoyer (1992). But this property alone seems to be too weak to be useful in practice although, of course, if this property is lacking it would definitely indicate 
a serious problem. To give a concrete example, the model-free, non-bootstrap interval $\left[\hat{D}_{x_{\mathrm{f}}}^{-1}(\alpha / 2), \hat{D}_{x_{\mathrm{f}}}^{-1}(1-\alpha / 2)\right]$ that Febrero-Bande proposes is asymptotically valid but it fails to take into account the variability that results from the error in estimating $D_{x_{\mathrm{f}}}$ by $\hat{D}_{x_{\mathrm{f}}}$. The result is a guaranteed under-coverage in finite samples. Without the assumption of Gaussian data, the bootstrap appears to be the only way to incorporate the estimator variability in the construction of a prediction interval.

In order to respond to the discussants in a unified and comprehensive way, I have collected some thoughts on asymptotic properties of prediction intervals in an Appendix. I have had the priviledge of running this short write-up by two authorities on asymptotics, Peter Hall and Anirban DasGupta, to whom I am grateful; all errors are, of course, mine.

I would like to end by reiterating my thanks to the discussants for their insightful contributions, and to the Editors, Ricardo Cao and Domingo Morales, for their invitation to present my work in Test and for their encouragement throughout the process.

\section{Appendix A: On asymptotic validity and correctness for pre- diction intervals}

Assume the data $\left\{\left(Y_{t}, x_{t}\right)\right.$ for $\left.t=1, \ldots, n\right\}$ follow the model of Section 3.1 in the paper, i.e., $Y_{t}=\mu\left(x_{t}\right)+\sigma\left(x_{t}\right) \varepsilon_{t}$, with $\varepsilon_{t}$ being i.i.d. $(0,1)$ from some distribution $F$. The regressor $x_{t}$ can be deterministic or random but independent of the error $\varepsilon_{k}$ for any $t$ and $k$. Our goal is to provide a prediction interval for $Y_{\mathrm{f}}$ that corresponds to a regressor value $X=x_{\mathrm{f}}$. Our predictive inference will be carried out conditionally on the regressors $x_{1}, \ldots, x_{n}$ and $x_{\mathrm{f}}$; so the discussion focuses on the notions of conditional validity and correctness to be defined shortly.

For example, consider the case of homoscedastic, linear regression, i.e., $\mu\left(x_{t}\right)=\beta_{0}+\beta_{1} x_{t}$,

and $\sigma\left(x_{t}\right)=\sigma$; their respective estimators are $\hat{\mu}\left(x_{t}\right)=\hat{\beta}_{0}+\hat{\beta}_{1} x_{t}$, and $S=\sqrt{(n-2)^{-1} \sum_{i=1}^{n} e_{i}^{2}}$ where $e_{i}=Y_{i}-\mu\left(x_{i}\right)$. Assuming further that the data are Gaussian, i.e., $F=\Phi$, the usual NORMAL theory $(1-\alpha) 100 \%$ prediction interval for $Y_{\mathrm{f}}$ is given by

$$
\hat{\mu}\left(x_{\mathrm{f}}\right) \pm t_{n-2}(\alpha / 2) S \sqrt{1+h_{\mathrm{f}}}
$$

where $h_{\mathrm{f}}=\underline{x}_{\mathrm{f}}^{\prime}\left(X^{\prime} X\right)^{-1} \underline{x}_{\mathrm{f}}$ is the 'leverage' at point $\underline{x}_{\mathrm{f}}=\left(1, x_{\mathrm{f}}\right)^{\prime}$, and $X$ is the design matrix.

Interval (A.1) is, of course, finite-sample correct under the normality assumption. For the sake of argument, however, consider also the following two prediction intervals:

$$
\hat{\mu}\left(x_{\mathrm{f}}\right) \pm z(\alpha / 2) S \sqrt{1+h_{\mathrm{f}}}
$$

and

$$
\hat{\mu}\left(x_{\mathrm{f}}\right) \pm z(\alpha / 2) S \text {. }
$$


Several researchers have been using the term asymptotically valid for prediction intervals whose coverage tends to the nominal asymptotically; see e.g., Schmoyer (1992). In this sense, both intervals (A.2) and (A.3) are asymptotically valid since $t_{n-2}(\alpha / 2) \rightarrow z(\alpha / 2)$ and $h_{\mathrm{f}} \rightarrow 0$ assuming, of course, that $\sum_{t=1}^{n} x_{t}^{2} \rightarrow \infty$ as $n \rightarrow \infty$.

Nevertheless, interval (A.3) can be called naive since it fails to take into account the variability that results from the error in estimating $\mu\left(x_{\mathrm{f}}\right)$ by $\hat{\mu}\left(x_{\mathrm{f}}\right)$, i.e., the leverage term $h_{\mathrm{f}}$. The result is that, although asymptotically valid, interval (A.3) will be characterized by under-coverage in finite samples; see Geisser (1993) for an in-depth discussion. Hence, asymptotic validity is perhaps too weak of a notion to be useful in practice although, of course, its absence would definitely indicate a serious problem.

By contrast, interval (A.2) does take account the variability resulting from estimation error. Since $t_{n-2}(\alpha / 2) \simeq z(\alpha / 2)$ as long as $n$ is reasonably large, interval (A.2) would be practically indistinguishable from the finite-sample correct interval (A.2) as long as $n$ is bigger than 50 or so. Therefore, interval (A.2) deserves to be called asymptotically correct.

To further complicate matters, consider the interval

$$
\hat{\mu}\left(x_{\mathrm{f}}\right) \pm z(\alpha / 2) S \sqrt{1+\lambda h_{\mathrm{f}}} \text { for some } \lambda \in(0,1)
$$

of which intervals (A.2) and (A.3) are limiting cases when $\lambda \rightarrow 1$ or 0 respectively. Interval (A.4) can then be called asymptotically valid and partially correct since it partially captures the variability resulting from estimation error.

Going back to the general model, assume that estimators $\hat{\mu}$ and $\hat{\sigma}$ are available that, as $n \rightarrow \infty$, satisfy

$$
\rho\left(\mathcal{L}\left(\tau_{n}(\hat{\mu}(x)-\mu(x))\right), J_{x}\right) \rightarrow 0 \text { and } \hat{\sigma}(x)=\sigma(x)+O_{P}\left(1 / n^{\zeta}\right)
$$

for all $x$ in an interval of interest, say $[A, B]$, and some $\zeta>0$; e.g., in the homoscedastic case where $\sigma(x)$ is constant, $\zeta=1 / 2$ under standard conditions. In the above, $\tau_{n} \rightarrow \infty$ as $n \rightarrow \infty, J_{x}$ is a non-degenerate distribution, $\mathcal{L}(Z)$ denotes the probability law of random variable $Z$, and $\rho(\cdot, \cdot)$ is a metric metrizing weak convergence of distributions.

To construct a prediction interval, we need an estimate of the distribution of the prediction root:

$$
Y_{\mathrm{f}}-\hat{\mu}\left(x_{\mathrm{f}}\right)=\left[\mu\left(x_{\mathrm{f}}\right)-\hat{\mu}\left(x_{\mathrm{f}}\right)\right]+\sigma\left(x_{\mathrm{f}}\right) \varepsilon_{\mathrm{f}}
$$

where $\varepsilon_{\mathrm{f}}=\left(Y_{\mathrm{f}}-\mu\left(x_{\mathrm{f}}\right)\right) / \sigma\left(x_{\mathrm{f}}\right)$; for concreteness, the $L_{2}$-optimal point predictor was used, i.e., the conditional expectation, whose estimated form coincides with $\hat{\mu}$.

Now consider a model-based, residual bootstrap algorithm that produces pseudo-data $\left\{\left(Y_{t}^{*}, x_{t}^{*}\right)\right.$ for $\left.t=1, \ldots, n\right\}$ by letting $Y_{t}^{*}=\hat{\mu}\left(x_{t}^{*}\right)+\hat{\sigma}\left(x_{t}^{*}\right) \varepsilon_{t}^{*}$ for $t=1, \ldots, n$, and also $Y_{\mathrm{f}}^{*}=\hat{\mu}\left(x_{\mathrm{f}}\right)+\hat{\sigma}\left(x_{\mathrm{f}}\right) \varepsilon_{\mathrm{f}}^{*}$, where $\varepsilon_{n}^{*}, \ldots, \varepsilon_{n}^{*}$ and $\varepsilon_{\mathrm{f}}^{*}$ are drawn i.i.d. from a distribution $F_{n}$. 
For example, in the $\mathrm{MB}$ and $\mathrm{MF} / \mathrm{MB}$ resampling algorithm of Section $3.5, F_{n}$ would be the empirical distribution function (e.d.f) of the (centered) residuals (fitted or predictive respectively), and $x_{t}=x_{t}^{*}$ for $t=1, \ldots, n$. For the large-sample success of the bootstrap, it is generally necessary that, as $n \rightarrow \infty$,

$$
F_{n} \stackrel{\mathcal{L}}{\Longrightarrow} F \text {. }
$$

If $F_{n}$ is the e.d.f. of the fitted residuals, condition (A.7) has been shown to hold by Akritas and Van Keilegom (2001); surprisingly, even when $\mu$ and $\sigma$ have to be estimated nonparametrically, $F_{n}$ maintains a $\sqrt{n}$ rate of convergence for $F$. Since the two types of residuals (fitted vs. predictive) coincide asymptotically, a similar result is expected to hold when $F_{n}$ is the e.d.f. of the predictive residuals. Note, however, that the algorithm could work with different choices of $F_{n}$ as long as they satisfy (A.7); for example, $F_{n}$ could be a smoothed version of the empirical distribution of the residuals.

Based on the bootstrap dataset $\left\{\left(Y_{t}^{*}, x_{t}^{*}\right)\right.$ for $\left.t=1, \ldots, n\right\}$, compute the bootstrap estimators $\hat{\mu}^{*}$ and $\hat{\sigma}^{*}$, and the bootstrap prediction root:

$$
Y_{\mathrm{f}}^{*}-\hat{\mu}^{*}\left(x_{\mathrm{f}}\right)=\left[\hat{\mu}\left(x_{\mathrm{f}}\right)-\hat{\mu}^{*}\left(x_{\mathrm{f}}\right)\right]+\hat{\sigma}\left(x_{\mathrm{f}}\right) \varepsilon_{\mathrm{f}}^{*} .
$$

As in Section 3.5, denote by $q(\alpha)$ the $\alpha$-quantile of the distribution of the above bootstrap prediction roots, and form the equal-tailed $(1-\alpha) 100 \%$ prediction interval

$$
\left[\hat{\mu}\left(x_{\mathrm{f}}\right)+q(\alpha / 2), \hat{\mu}\left(x_{\mathrm{f}}\right)+q(1-\alpha / 2)\right] .
$$

Good properties of bootstrap prediction (and confidence) intervals hinge on the bootstrap being able to mimic well the distributions of estimators of interest. For example, it is important to be able to prove that, as $n \rightarrow \infty$,

$$
\rho\left(\mathcal{L}^{*}\left(\tau_{n}\left(\hat{\mu}^{*}(x)-\hat{\mu}(x)\right)\right), J_{x}\right) \stackrel{P}{\longrightarrow} 0
$$

for all $x$ in $[A, B]$; here, $\mathcal{L}^{*}\left(Z^{*}\right)$ denotes the probability law of quantity $Z^{*}$ in the bootstrap world, and $J_{x}$ is the same distribution as in eq. (A.5).

Remark A.1 Coupled with eq. (A.5), eq. (A.10) is just the condition guaranteeing the asymptotic validity of confidence intervals based on the above model-based bootstrap. If $\mu$ belongs to a parametric family, e.g., linear regression, things are straightforward. In a nonparametric setting, however, there are some intricacies due to bias. To elaborate, condition (A.10) has been verified by several authors under the nonparametric regression model of Section 3.1; see e.g. Neumann and Polzehl (1998) and the references therein. Typically, $J_{x}$ is $N\left(B_{x}, V_{x}^{2}\right)$ where $V_{x}^{2}$ is given in eq. (25) of the paper. The bias term $B_{x}$ is more problematic; the easiest way to handle it is via an under-smoothing bandwidth 
choice, i.e., a choice of bandwidth that makes the bias of $\hat{\mu}(x)$ and $\hat{\mu}^{*}(x)$ to be of smaller order than their respective standard deviation - see Hall (1992). Doing this implies that the limiting bias is zero in eq. (A.5) as well as (A.10), i.e., $B_{x}=0$. Without an under-smoothed bandwidth, the interval (A.9) must be explicitly corrected for bias and/or two different bandwidths must be used; these options will be re-visited later on.

Remark A.2 The application of eq. (A.5) and (A.10) to either confidence or prediction intervals, hinges on looking at these large-sample distributions for $x=x_{\mathrm{f}}$. If eq. (A.5) and (A.10) are valid for all $x$ in $[A, B]$, then it better be the case that $x_{\mathrm{f}}$ belongs to $[A, B]$ as well. In the nonparametric case, the interval $[A, B]$ would necessarily be a subset of the convex hull of the data points $x_{1}, \ldots, x_{n}$. Furthermore, the points $x_{1}, \ldots, x_{n}$ should be 'regularly' (and densely) distributed over $[A, B]$ either by design, or by them being the outcome of a random sample whose density over $[A, B]$ is regular (and positive); see the discussion just before eq. (25) of the paper. To give a counterexample, if $x_{\mathrm{f}}$ were to be further than a bandwidth away from the convex hull of $x_{1}, \ldots, x_{n}$, then prediction and/or confidence statements would be vacuous; technically, this would be the case where eq. (A.5) and (A.10) are not valid because $J_{x_{\mathrm{f}}}$ degenerates to a point mass at zero.

The question now is how to quantify the accuracy of a prediction interval. To start, consider the prediction root of eq. (A.6). Since $\tau_{n} \rightarrow \infty$, eq. (A.10) implies that the term $Z_{n}=\hat{\mu}\left(x_{\mathrm{f}}\right)-\mu\left(x_{\mathrm{f}}\right)$ tends to 0 in probability, and is thus asymptotically negligible. Similarly, eq. (A.10) implies that the term $Z_{n}^{*}=\hat{\mu}^{*}\left(x_{\mathrm{f}}\right)-\hat{\mu}\left(x_{\mathrm{f}}\right)$ is asymptotically negligible in the bootstrap root of eq. (A.8). So why not omit these two terms, and thus construct a bootstrap prediction interval simply based on the empirical distribution of the residuals, i.e., based on the quantiles of $\hat{\sigma}\left(x_{\mathrm{f}}\right) \varepsilon_{\mathrm{f}}^{*}$ ? This would indeed have the desired coverage level asymptotically due to eq. (A.7) and the RHS of eq. (A.10). Furthermore, one would not need the bootstrap to calculate this prediction interval as the quantiles of $\varepsilon_{\mathrm{f}}^{*}$ are simply the quantiles of $F_{n}$. In fact, this simplified proposal has been considered the literature; it is the prediction interval of eq. (2) of Akritas and Van Keilegom (2001).

The reason that the above simplified proposal is not recommended is that it fails to incorporate the variability of the estimated quantities on the prediction interval; the uncertainty due to estimation error should result into wider intervals compared to the case where the neglected terms are exactly zero. However, as mentioned above, the limiting coverage is unaffected; thus, similarly to the naive interval (A.3), the simplified proposal leads to a prediction interval that is asymptotically valid but not correct. By the same token, the model-based interval (A.9) is also asymptotically valid - but is it correct?

To quantify this finite-sample effect of the terms $Z_{n}$ and $Z_{n}^{*}$ on the coverage probabilities, we may try to construct an asymptotic expansion. In what follows we will assume that the 
error distribution $F$ is twice continuously differentiable with $f=F^{\prime}$. Denote the distribution of $Z_{n}$ by $G_{n}(z)=P\left\{Z_{n} \leq z\right\}$. For ease of notation, denote by $F_{\sigma}$ and $f_{\sigma}$ the distribution and density of $\sigma\left(x_{\mathrm{f}}\right) \cdot \varepsilon_{\mathrm{f}}$, i.e., $F_{\sigma}(y)=F\left(y / \sigma\left(x_{\mathrm{f}}\right)\right)$. Using the independence between $\varepsilon_{\mathrm{f}}$ and $Z_{n}$, we can approximate the distribution of the prediction root of eq. (A.6). In the formula below, the probability $P$ is to be interpreted as conditional on $x_{1}, \ldots, x_{n}$ and $x_{\mathrm{f}}$ although the conditioning will not be explicitly denoted.

$$
\begin{gathered}
P\left\{Y_{\mathrm{f}}-\hat{\mu}\left(x_{\mathrm{f}}\right) \leq y\right\}=P\left\{\sigma\left(x_{\mathrm{f}}\right) \varepsilon_{\mathrm{f}} \leq y+Z_{n}\right\}=\int_{-\infty}^{\infty} P\left\{\sigma\left(x_{\mathrm{f}}\right) \varepsilon_{\mathrm{f}} \leq y+z \mid Z_{n}=z\right\} d G_{n}(z) \\
=\int F_{\sigma}(y+z) d G_{n}(z)=\int\left(F_{\sigma}(y)+f_{\sigma}(y) \cdot z+\frac{f_{\sigma}^{\prime}(y)}{2} \cdot z^{2}+o\left(z^{2}\right)\right) d G_{n}(z) \\
=F_{\sigma}(y)+f_{\sigma}(y) \cdot E Z_{n}+\frac{f_{\sigma}^{\prime}(y)}{2} \cdot E Z_{n}^{2}+o\left(E Z_{n}^{2}\right)
\end{gathered}
$$

where the above Taylor approximation is justified from the fact that $Z_{n} \stackrel{P}{\longrightarrow} 0$. Note that $E Z_{n}=\operatorname{Bias}\left(\hat{\mu}\left(x_{\mathrm{f}}\right)\right)$ and $E Z_{n}^{2}=E\left(\hat{\mu}\left(x_{\mathrm{f}}\right)-\mu\left(x_{\mathrm{f}}\right)\right)^{2}$, i.e., the Mean Squared Error (MSE) of estimator $\hat{\mu}\left(x_{\mathrm{f}}\right)$.

A higher-order accurate prediction interval would be based on an approximation of the distribution of the prediction root $Y_{\mathrm{f}}-\hat{\mu}\left(x_{\mathrm{f}}\right)$ that captures the first three terms of expansion of eq. (A.11). Can the bootstrap yield higher-order accuracy? To investigate, let us create the analog of eq. (A.11) in the bootstrap world under the assumption that $F_{n}$ is twice continuously differentiable with $f_{n}=F_{n}^{\prime}$; e.g., if the e.d.f. of the residuals has to be used, it has to smoothed beforehand. As before, let $F_{n, \hat{\sigma}}$ and $f_{n, \hat{\sigma}}$ denote the distribution and density of $\hat{\sigma}\left(x_{\mathrm{f}}\right) \varepsilon_{\mathrm{f}}^{*}$ in the bootstrap world, i.e., $F_{n, \hat{\sigma}}(y)=F_{n}\left(y / \hat{\sigma}\left(x_{\mathrm{f}}\right)\right)$. Similar arguments yield:

$$
P^{*}\left\{Y_{\mathrm{f}}^{*}-\hat{\mu}^{*}\left(x_{\mathrm{f}}\right) \leq y\right\}=F_{n, \hat{\sigma}}(y)+f_{n, \hat{\sigma}}(y) E^{*} Z_{n}^{*}+\frac{f_{n, \hat{\sigma}}^{\prime}(y)}{2} E^{*}\left(Z_{n}^{*}\right)^{2}+o_{P}\left(E^{*}\left(Z_{n}^{*}\right)^{2}\right)
$$

which - at least formally - appears to imitate the expansion (A.11). By contrast, the simplified prediction interval of eq. (2) of Akritas and Van Keilegom (2001) is based on approximating (A.11) by $F_{n, \hat{\sigma}}(y)$, i.e., just the first term in the above.

In the paper, the possibility of obtaining prediction intervals via approximating the distribution of the studentized root $\left(Y_{\mathrm{f}}-\hat{\mu}\left(x_{\mathrm{f}}\right)\right) / \hat{\sigma}\left(x_{\mathrm{f}}\right)$ by the bootstrap distribution of the studentized root $\left(Y_{\mathrm{f}}^{*}-\hat{\mu}^{*}\left(x_{\mathrm{f}}\right)\right) / \hat{\sigma}^{*}\left(x_{\mathrm{f}}\right)$ is also mentioned. Note that for the studentized bootstrap to work, we would additionally need that

$$
\rho\left(\mathcal{L}^{*}\left(\hat{\sigma}^{*}(x)\right), \delta_{\sigma(x)}\right) \stackrel{P}{\longrightarrow} 0
$$


for all $x \in[A, B]$ as $n \rightarrow \infty$, where $\delta_{\sigma(x)}$ denotes a point mass ${ }^{1}$ at $\sigma(x)$. The above, of course, follows from eq. (A.5) if (say) $E^{*}\left(\hat{\sigma}^{*}(x)-\hat{\sigma}(x)\right)^{2} \stackrel{P}{\longrightarrow} 0$.

In the studentized case, the general expansions (A.11) and (A.12) would have to be re-worked as follows:

$$
\begin{gathered}
P\left\{\frac{Y_{\mathrm{f}}-\hat{\mu}\left(x_{\mathrm{f}}\right)}{\hat{\sigma}\left(x_{\mathrm{f}}\right)} \leq y\right\}=\tilde{F}(y)+\tilde{f}(y) \cdot E Z_{n}+\frac{\tilde{f}^{\prime}(y)}{2} \cdot E Z_{n}^{2}+o\left(E Z_{n}^{2}\right), \\
P^{*}\left\{\frac{Y_{\mathrm{f}}^{*}-\hat{\mu}^{*}\left(x_{\mathrm{f}}\right)}{\hat{\sigma}^{*}\left(x_{\mathrm{f}}\right)} \leq y\right\}=\tilde{F}_{n}(y)+\tilde{f}_{n}(y) E^{*} Z_{n}^{*}+\frac{\tilde{f}_{n}^{\prime}(y)}{2} E^{*}\left(Z_{n}^{*}\right)^{2}+o_{P}\left(E^{*}\left(Z_{n}^{*}\right)^{2}\right)
\end{gathered}
$$

where $\tilde{F}$ and $\tilde{f}$ denote the distribution and density of $\sigma\left(x_{\mathrm{f}}\right) \varepsilon_{\mathrm{f}} / \hat{\sigma}\left(x_{\mathrm{f}}\right)$; note that $\hat{\sigma}$ is a function of $\varepsilon_{1}, \ldots, \varepsilon_{n}$ and thus independent of $\varepsilon_{\mathrm{f}}$. Similarly, $\tilde{F}_{n}$ and $\tilde{f}_{n}$ denote the distribution and density of $\hat{\sigma}\left(x_{\mathrm{f}}\right) \varepsilon_{\mathrm{f}}^{*} / \hat{\sigma}^{*}\left(x_{\mathrm{f}}\right)$ in the bootstrap world. As a result, the equal-tailed $(1-\alpha) 100 \%$ studentized prediction interval would be given by

$$
\left[\hat{\mu}\left(x_{\mathrm{f}}\right)+q^{\prime}(\alpha / 2) \hat{\sigma}\left(x_{\mathrm{f}}\right), \hat{\mu}\left(x_{\mathrm{f}}\right)+q^{\prime}(1-\alpha / 2) \hat{\sigma}\left(x_{\mathrm{f}}\right)\right]
$$

where $q^{\prime}(\alpha)$ is the $\alpha$-quantile of distribution (A.15).

For example, under the assumption (and knowledge) that $F=\Phi$, the NORMAL theory interval (A.1) for linear regression matches exactly the first three terms of eq. (A.14) with $\tilde{F}$ being the $t_{n-2}$ distribution, $E Z_{n}=0$, and no remainder term. Without Gaussianity, resampling methods seem to give the only hope of producing accurate prediction intervals.

Consider the following cases:

- $\mu$ belongs to a parametric family. In this case, we typically have $E Z_{n}=O(1 / n)$ and $E Z_{n}^{2}=O(1 / n)$.

- $F$ is known. In this case, of course, we would let $F_{n}=F$. But even in this rather unrealistic case, expansion (A.12) can not match (A.11) because $F_{n, \hat{\sigma}}(y)$ is not accurate enough. For example, even when $\hat{\sigma}(x)=\sigma(x)+O_{P}(1 / \sqrt{n})$, we would have $F_{n, \hat{\sigma}}(y)=F_{\sigma}(y)+O_{P}(1 / \sqrt{n})$; thus, terms of order $O(1 / n)$ would not be captured. However, the studentized bootstrap would be higher-order accurate here. Letting $F_{n}=F$, expansion (A.15) will match up to the first three terms of (A.14) implying that the studentized interval (A.16) is higher-order accurate. Thus, the studentized bootstrap extends the availability of higher-order accurate

\footnotetext{
${ }^{1}$ An additional assumption is also needed for our prediction interval application, namely that $\sigma(x)>0$ for all $x$ in a neighborhood of $x_{\mathrm{f}}$; of course, if this is assumption is violated, then this is actually good news since (unstudentized) prediction can be made without error.
} 
prediction intervals from the Gaussian case to the case of any known (smooth) distribution $F$.

- $\mathbf{F}$ is unknown. Even if $F$ is assumed to belong to a parametric family, the error in estimating $F$ by the (parametric) estimator $F_{n}$ will not be smaller than $O_{P}(1 / \sqrt{n})$. Not even studentization can help achieve higher-order accuracy since the first term of the expansion (A.15) will differ by $O_{P}(1 / \sqrt{n})$ from the first term of (A.14).

- $\mu$ belongs to a nonparametric family, e.g., a smoothness class. In this case, $E Z_{n}^{2}=O\left(\tau_{n}^{-2}\right)=O\left(1 / n^{1-\delta}\right)$ for some $\delta>0$. If $\hat{\mu}$ is a kernel estimator, then this implies the bandwidth choice $h \sim 1 / n^{\delta}$ since $\tau_{n}=\sqrt{n h}$. The undersmoothing condition ${ }^{2}$ discussed in Remark A.1 would imply $E Z_{n}=\operatorname{Bias}\left(\hat{\mu}\left(x_{\mathrm{f}}\right)\right)=o\left(\tau_{n}^{-1}\right)=o\left(\left(1 / n^{(1-\delta) / 2}\right)\right.$. In general, suppose the exact order of magnitude of $E Z_{n}=\operatorname{Bias}\left(\hat{\mu}\left(x_{\mathrm{f}}\right)\right)$ is given by $O\left(1 / n^{\gamma}\right)$ for some $\gamma>(1-\delta) / 2$. The problem here is that although the bootstrap will match the MSE term well, it might not match the bias term perfectly. In other words, we will typically have

$$
n^{1-\delta}\left(E Z_{n}^{2}-E^{*}\left(Z_{n}^{*}\right)^{2}\right)=o_{P}(1) \text { but } n^{\gamma}\left(E Z_{n}-E^{*} Z_{n}^{*}\right) \neq o_{P}(1) .
$$

Hence, the bootstrap prediction intervals (studentized or not) will not be higher order accurate despite the fact that we can have an estimator $F_{n}$ that is $\sqrt{n}$-consistent for $F$ as in Akritas and Van Keilegom (2001).

The asymptotic expansion results are inconclusive in the nonparametric case but some insights are available.

(a) Although the terms $Z_{n}$ and $Z_{n}^{*}$ are asymptotically negligible, it is not recommended to set them to zero in the construction of prediction intervals.

(b) The model-based bootstrap is not expected to precisely quantify the bias term in the expansion but the bootstrap estimate of bias will still be a correction in the right direction, e.g., if $\operatorname{Bias}\left(\hat{\mu}\left(x_{\mathrm{f}}\right)\right)$ is big and positive, so will $\operatorname{Bias}^{*}\left(\hat{\mu}^{*}\left(x_{\mathrm{f}}\right)\right)$; this can make a difference in the finite-sample practical setting.

(c) The model-based bootstrap is expected to capture the 3rd term in the aforementioned expansions, e.g., the MSE term.

\footnotetext{
${ }^{2}$ The choice of $\delta$ in the above has to do with the smoothness of $\mu$ and the estimation method used; e.g., the order of the kernel when kernel smoothing is used. For example, assuming that $\mu$ is twice continuously differentiable, undersmoothing requires $\delta>1 / 5$ when a 2 nd order kernel is used, in which case $E Z_{n}=$ $O\left(h^{2}\right)=O\left(n^{-2 \delta}\right)$.
} 
The above justify applying the term 'asymptotically valid and partially correct' to the model-based intervals by analogy to interval (A.4). Of course, the model-based bootstrap construction would give the desired results only under the asymptotic conditions that have been previously discussed. We collect them below for easy reference.

Fact A. Under the stated additive model and estimation methodology including condition (A.5), the model-based bootstrap prediction interval (A.9) will be an asymptotically valid and partially correct $(1-\alpha) 100 \%$ prediction interval for $Y_{\mathrm{f}}$ provided that eq. (A.7) and (A.10) hold true. If in addition, eq. (A.13) holds, the studentized interval (A.16) will also be an asymptotically valid and partially correct $(1-\alpha) 100 \%$ prediction interval for $Y_{\mathrm{f}}$.

Therefore, under these standard regularity conditions, both MB and MF/MB intervals of Section 3.5 are asymptotically valid and partially correct.

To intuitively appreciate the statement of Fact A, focus on the unstudentized case, i.e., interval (A.9), and note that the distribution of the prediction root of eq. (A.6) isdue to independence - approximately given by $\tau_{n}^{-1} J_{x_{\mathrm{f}}} \star F_{\sigma}$ where $\star$ denotes convolution. Similarly, the distribution of the bootstrap prediction root of eq. (A.8) is approximately given by $\tau_{n}^{-1} J_{x_{\mathrm{f}}} \star F_{n, \hat{\sigma}}$ which is close to $\tau_{n}^{-1} J_{x_{\mathrm{f}}} \star F_{\sigma}$. So the bootstrap distribution seems to approximately capture the distortion to $F_{\sigma}$ caused by convolving it with the factor $\tau_{n}^{-1} J_{x_{\mathrm{f}}}$ that is the result of the estimation error; hence, the asymptotic (partial) correctness of the resulting prediction interval is justified.

Finally, recall that we have focused on the popular under-smoothing condition of Remark A.1 where it was noted that two alternatives exist in order to address the bias issue. These proposals were originally developed for confidence intervals by Härdle and Bowman (1988), and Härdle and Marron (1991) respectively; they are re-formulated below specifically for the prediction interval application.

- Use an explicit correction for the bias. To fix ideas, assume that $\mu$ is twice continuously differentiable, and a 2nd order smoothing kernel is used. The correction amounts to replacing the (unstudentized) bootstrap prediction root $Y_{\mathrm{f}}^{*}-\hat{\mu}^{*}\left(x_{\mathrm{f}}\right)$ of eq. (A.8) by the bias-corrected prediction root $Y_{\mathrm{f}}^{*}-E^{*} \hat{\mu}^{*}\left(x_{\mathrm{f}}\right)+\hat{\mu}^{\prime \prime}\left(x_{\mathrm{f}}\right) h^{2} / 2$. Letting $q^{\prime \prime}(\alpha)$ denote the $\alpha$-quantile of the distribution of the above bias-corrected prediction root, the resulting equal-tailed, $(1-\alpha) 100 \%$ prediction interval is given by

$$
\left[\hat{\mu}\left(x_{\mathrm{f}}\right)+q^{\prime \prime}(\alpha / 2), \hat{\mu}\left(x_{\mathrm{f}}\right)+q^{\prime \prime}(1-\alpha / 2)\right] .
$$

- Use an over-smoothed bandwidth in creating the $Y_{t}^{*}$ in the bootstrap world. In other words, let $Y_{t}^{*}=\tilde{\mu}\left(x_{t}^{*}\right)+\hat{\sigma}\left(x_{t}^{*}\right) \varepsilon_{t}^{*}$ where $\tilde{\mu}$ is computed in the same way as $\hat{\mu}$ but using a larger bandwidth (by an order of magnitude); the rest of the algorithm remains as stated. 
Employing either of the above would allow the practitioner to use the MSE-optimal bandwidth for $\hat{\mu}$-and not resort to under-smoothing - while making the bootstrap estimate of bias match the real-world bias to the correct order, i.e., making sure that the RHS of (A.17) is indeed $o_{P}(1)$. The last statement is particularly important because in this case, the main difference between the real-world expansion (A.11) and the bootstrap expansion (A.12) would be the difference between $F_{n, \hat{\sigma}}(y)$ and $F_{\sigma}(y)$ (and their respective derivatives). If $\hat{\sigma}(x)=\sigma(x)+O_{P}(1 / \sqrt{n})$, e.g., in the homoscedastic case, the aforementioned $\sqrt{n}$ - convergence of $F_{n}$ to $F$ would imply that $F_{n, \hat{\sigma}}(y)=F_{\sigma}(y)+O_{P}(1 / \sqrt{n})$. Hence, the bootstrap expansion (A.12) would appear to match the first two terms of expansion (A.11), further justifying the label 'partially correct' for the resulting prediction intervals. More work is required to fully realize the practical benefit of the above proposals in the construction of prediction intervals.

\section{References}

[1] Akritas, M.G., and Van Keilegom, I. (2001). Non-parametric estimation of the residual distribution, Scand. J. Statist., vol. 28, no. 3, pp. 549-567.

[2] Cai, T.T., and Hall, P. (2006). Prediction in functional linear regression, Ann. Statist., vol. 35, no. 5, pp. 2159-2179.

[3] Ferraty, F., and Vieu, P. (2006). Nonparametric Functional Data Analysis: Theory and Practice, Springer, New York.

[4] Hall, P. (1992). On bootstrap confidence intervals in nonparametric regression, Ann. Statist., vol. 20, no. 2, pp. 695-711.

[5] Härdle, W. and Bowman, A.W. (1988). Bootstrapping in nonparametric regression: local adaptive smoothing and confidence bands, J. Amer. Statist. Assoc., vol. 83, no. 401, pp. 102-110.

[6] Härdle, W. and Marron, J.S. (1991). Bootstrap simultaneous error bars for nonparametric regression, Ann. Statist., vol. 19, no. 2, pp. 778-796.

[7] Paparoditis, E., and Politis, D.N. (1999). The local bootstrap for periodogram statistics, J. Time Ser. Anal., vol. 20, no. 2, pp. 193-222. 